COMPUTATIONAL METHODS IN APPLIED MATHEMATICS, Vol.5(2005), No. 1, pp. 97-104

(C) 2005 Institute of Mathematics of the National Academy of Sciences of Belarus

\title{
GENERALIZATION OF THE CORRECTED MID-POINT RULE AND ERROR BOUNDS
}

\author{
NENAD UJEVIĆ \\ Department of Mathematics, University of Split \\ Teslina 12/III, 21000 Split, Croatia
}

\begin{abstract}
A generalization of the corrected mid-point rule is derived. Various error bounds for this generalization are established.
\end{abstract}

2000 Mathematics Subject Classification: 65D30; 26D15.

Keywords: quadrature rule, generalization, numerical integration, error bounds.

\section{Introduction}

In recent years a number of authors have considered error inequalities for some known and some new quadrature rules. Sometimes they have considered generalizations of these rules. For example, the well-known mid-point and corrected mid-point quadrature rules are considered in $[1,2,7-9]$ and some generalizations are given in [1] and [2]. In [2] we can find

$$
\int_{a}^{b} f(t) d t=\sum_{k=0}^{n-1}\left[1+(-1)^{k}\right] \frac{(b-a)^{k+1}}{2^{k+1}(k+1) !} f^{(k)}\left(\frac{a+b}{2}\right)+(-1)^{n} \int_{a}^{b} K_{n}(t) f^{(n)}(t) d t
$$

where

$$
K_{n}(t)= \begin{cases}\frac{(t-a)^{n}}{n !}, & t \in\left[a, \frac{a+b}{2}\right] \\ \frac{(t-b)^{n}}{n !}, & t \in\left(\frac{a+b}{2}, b\right] .\end{cases}
$$

For $n=1$ we get the mid-point rule

$$
\int_{a}^{b} f(t) d t=(b-a) f\left(\frac{a+b}{2}\right)-\int_{a}^{b} K_{1}(t) f^{\prime}(t) d t
$$

In this paper we consider the generalization of the corrected mid-point rule and give corresponding error inequalities. Similar error inequalities are established in [9] and [10]. We also give a numerical example which shows that this generalization can be very effective. 


\section{Main results}

Theorem 2.1. Let $f:[a, b] \rightarrow R$ be a function such that $f^{(n-1)}$ is absolutely continuous. Then

$$
\begin{aligned}
\int_{a}^{b} f(x) d x= & f\left(\frac{a+b}{2}\right)(b-a)+\frac{f^{\prime}(b)-f^{\prime}(a)}{24}(b-a)^{2} \\
& -\frac{1}{6} \sum_{i=2}^{m} \frac{(b-a)^{2 i+1}(2 i-2)(2 i+3)}{2^{2 i}(2 i+1) !} f^{(2 i)}\left(\frac{a+b}{2}\right)+R(f),
\end{aligned}
$$

where $m=\left[\frac{n-1}{2}\right]$, the integer part of $(n-1) / 2$,

$$
R(f)=(-1)^{n} \int_{a}^{b} S_{n}(t) f^{(n)}(t) d t
$$

and

$$
S_{n}(t)= \begin{cases}\frac{(t-a)^{n-2}}{n !}\left[(t-a)^{2}-\frac{n(n-1)}{24}(b-a)^{2}\right], & t \in\left[a, \frac{a+b}{2}\right], \\ \frac{(t-b)^{n-2}}{n !}\left[(t-b)^{2}-\frac{n(n-1)}{24}(b-a)^{2}\right], & t \in\left(\frac{a+b}{2}, b\right] .\end{cases}
$$

Proof. We prove (1) by induction. First we note that

$$
\begin{aligned}
& S_{2}(t)= \begin{cases}\frac{1}{2}(t-a)^{2}-\frac{1}{24}(b-a)^{2}, & t \in\left[a, \frac{a+b}{2}\right], \\
\frac{1}{2}(t-b)^{2}-\frac{1}{24}(b-a)^{2}, & t \in\left(\frac{a+b}{2}, b\right],\end{cases} \\
& S_{3}(t)= \begin{cases}\frac{(t-a)}{3 !}\left[(t-a)^{2}-\frac{1}{4}(b-a)^{2}\right], & t \in\left[a, \frac{a+b}{2}\right], \\
\frac{(t-b)}{3 !}\left[(t-b)^{2}-\frac{1}{4}(b-a)^{2}\right], & t \in\left(\frac{a+b}{2}, b\right],\end{cases} \\
& S_{4}(t)= \begin{cases}\frac{(t-a)^{2}}{4 !}\left[(t-a)^{2}-\frac{1}{2}(b-a)^{2}\right], & t \in\left[a, \frac{a+b}{2}\right], \\
\frac{(t-b)^{2}}{4 !}\left[(t-b)^{2}-\frac{1}{2}(b-a)^{2}\right], & t \in\left(\frac{a+b}{2}, b\right]\end{cases}
\end{aligned}
$$

are the Peano kernels for the corrected mid-point quadrature rule, that is, we have

$$
\begin{aligned}
\int_{a}^{b} S_{4}(t) f^{(4)}(t) d t & =-\int_{a}^{b} S_{3}(t) f^{\prime \prime \prime}(t) d t=\int_{a}^{b} S_{2}(t) f^{\prime \prime}(t) d t \\
& =-\frac{f^{\prime}(b)-f^{\prime}(a)}{24}(b-a)^{2}-f\left(\frac{a+b}{2}\right)(b-a)+\int_{a}^{b} f(t) d t .
\end{aligned}
$$

We easily show that (1) holds for $n=5$. Now suppose that (1) holds for an arbitrary $n$. We have to prove that (1) holds for $n \rightarrow n+1$. To simplify the proof, we introduce the notations

$$
\begin{aligned}
& P_{n}(t)=\frac{(t-a)^{n-2}}{n !}\left[(t-a)^{2}-\frac{n(n-1)}{24}(b-a)^{2}\right], \\
& Q_{n}(t)=\frac{(t-b)^{n-2}}{n !}\left[(t-b)^{2}-\frac{n(n-1)}{24}(b-a)^{2}\right] .
\end{aligned}
$$


We see that $P_{n}$ and $Q_{n}$ form Appell sequences of polynomials, that is

$$
P_{n}^{\prime}(t)=P_{n-1}(t), \quad Q_{n}^{\prime}(t)=Q_{n-1}(t) .
$$

Then we have

$$
\begin{aligned}
& (-1)^{n+1} \int_{a}^{b} S_{n+1}(t) f^{(n+1)}(t) d t \\
& =(-1)^{n+1} \int_{a}^{\frac{a+b}{2}} P_{n+1}(t) f^{(n+1)}(t) d t+(-1)^{n+1} \int_{\frac{a+b}{2}}^{b} Q_{n+1}(t) f^{(n+1)}(t) d t \\
& =(-1)^{n+1}\left[P_{n+1}\left(\frac{a+b}{2}\right) f^{(n)}\left(\frac{a+b}{2}\right)-P_{n+1}(a) f^{(n)}(a)\right]+(-1)^{n} \int_{a}^{\frac{a+b}{2}} P_{n}(t) f^{(n)}(t) d t \\
& +(-1)^{n+1}\left[Q_{n+1}(b) f^{(n)}(b)-Q_{n+1}\left(\frac{a+b}{2}\right) f^{(n)}\left(\frac{a+b}{2}\right)\right]+(-1)^{n} \int_{\frac{a+b}{2}}^{b} Q_{n}(t) f^{(n)}(t) d t \\
& =(-1)^{n} \int_{a}^{b} S_{n}(t) f^{(n)}(t) d t+(-1)^{n+1}\left[P_{n+1}\left(\frac{a+b}{2}\right)-Q_{n+1}\left(\frac{a+b}{2}\right)\right] f^{(n)}\left(\frac{a+b}{2}\right) \\
& =-f\left(\frac{a+b}{2}\right)(b-a)-\frac{f^{\prime}(b)-f^{\prime}(a)}{24}(b-a)^{2}+\int_{a}^{b} f(t) d t \\
& +\frac{1}{6} \sum_{i=2}^{m} \frac{(b-a)^{2 i+1}(2 i-2)(2 i+3)}{2^{2 i}(2 i+1) !} f^{(2 i)}\left(\frac{a+b}{2}\right) \\
& +(-1)^{n+1}\left[P_{n+1}\left(\frac{a+b}{2}\right)-Q_{n+1}\left(\frac{a+b}{2}\right)\right] f^{(n)}\left(\frac{a+b}{2}\right) \\
& =-f\left(\frac{a+b}{2}\right)(b-a)-\frac{f^{\prime}(b)-f^{\prime}(a)}{24}(b-a)^{2}+\int_{a}^{b} f(t) d t \\
& +\frac{1}{6} \sum_{i=2}^{m_{1}} \frac{(b-a)^{2 i+1}(2 i-2)(2 i+3)}{2^{2 i}(2 i+1) !} f^{(2 i)}\left(\frac{a+b}{2}\right),
\end{aligned}
$$

where $m_{1}=\left[\frac{n}{2}\right]$, since

$$
\begin{aligned}
(-1)^{n+1} & {\left[P_{n+1}\left(\frac{a+b}{2}\right)-Q_{n+1}\left(\frac{a+b}{2}\right)\right] f^{(n)}\left(\frac{a+b}{2}\right) } \\
& =(-1)^{n} \frac{(b-a)^{n+1}}{2^{n+1}(n+1) !}\left[1-(-1)^{n+1}\right] \frac{(n+3)(n-2)}{6} f^{(n)}\left(\frac{a+b}{2}\right) .
\end{aligned}
$$

This completes the proof. 
Lemma 2.1. The Peano kernels $S_{n}(t), n>3$, satisfy

$$
\begin{aligned}
\int_{a}^{b} S_{n}(t) d t & =0, \text { if } n \text { is odd, } \\
\int_{a}^{b}\left|S_{n}(t)\right| d t & =\frac{(n+3)(n-2)}{3} \frac{(b-a)^{n+1}}{2^{n+1}(n+1) !}, \\
\max _{t \in[a, b]}\left|S_{n}(t)\right| & =\frac{(n-3)(n+2)}{3} \frac{(b-a)^{n}}{2^{n+1} n !} .
\end{aligned}
$$

Proof. A simple calculation gives

$$
\int_{a}^{b} S_{n}(t) d t=\frac{(b-a)^{n+1}}{2^{n+1}(n+1) !}\left[1-(-1)^{n+1}\right]\left[1-\frac{n(n+1)}{6}\right] .
$$

From the above relation we see that (6) holds, since $1-(-1)^{n+1}=0$ if $n$ is odd.

We now consider some properties of the Appell sequences of polynomials $P_{n}(t)$ and $Q_{n}(t)$, given by (4) and (5), respectively. We have that $(t-a)^{n-2} \geqslant 0$ for each $n>3$ and $t \in\left[a, \frac{a+b}{2}\right]$. We also have

$$
(t-a)^{2}-\frac{n(n-1)}{24}(b-a)^{2} \leqslant 0, \quad t \in\left[a, \frac{a+b}{2}\right], \quad n>3,
$$

such that $P_{n}(t) \leqslant 0$ for $n>3$ and $t \in\left[a, \frac{a+b}{2}\right]$. Since $P_{n}^{\prime}(t)=P_{n-1}(t)$, we conclude that $P_{n}(t)$ are decreasing functions. Since

We have that $(t-b)^{n-2} \leqslant 0$ if $n>3, n$ is odd and $(t-b)^{n-2} \geqslant 0$ if $n>3, n$ is even.

$$
(t-b)^{2}-\frac{n(n-1)}{24}(b-a)^{2} \leqslant 0, \quad t \in\left[\frac{a+b}{2}, b\right], \quad n>3,
$$

we have that $Q_{n}(t) \leqslant 0$ if $n$ is even and $Q_{n}(t) \geqslant 0$ if $n$ is odd. As we know, $Q_{n}^{\prime}(t)=Q_{n-1}(t)$ such that $Q_{n}(t)$ are decreasing functions if $n$ is odd and $Q_{n}(t)$ are increasing functions if $n$ is even. We use these properties to prove (7) and (8).

We have

$$
\begin{aligned}
\int_{a}^{b}\left|S_{n}(t)\right| d t & =\int_{a}^{\frac{a+b}{2}}\left|P_{n}(t)\right| d t+\int_{\frac{a+b}{2}}^{b}\left|Q_{n}(t)\right| d t \\
& =\left|\int_{a}^{\frac{a+b}{2}} P_{n}(t) d t\right|+\left|\int_{\frac{a+b}{2}}^{b} Q_{n}(t) d t\right| \\
& =\frac{1}{3} \frac{\left(n^{2}+n-6\right)(b-a)^{n+1}}{2^{n+1}(n+1) !} .
\end{aligned}
$$


Finally, we have

$$
\begin{aligned}
\max _{t \in[a, b]}\left|S_{n}(t)\right| & =\max \left\{\max _{t \in\left[a, \frac{a+b}{2}\right]}\left|P_{n}(t)\right|, \max _{t \in\left[\frac{a+b}{2}, b\right]}\left|Q_{n}(t)\right|\right\} \\
& =\max \left\{\left|P_{n}\left(\frac{a+b}{2}\right)\right|,\left|Q_{n}\left(\frac{a+b}{2}\right)\right|\right\} \\
& =\frac{1}{3} \frac{\left(n^{2}-n-6\right)(b-a)^{n}}{2^{n+1} n !} .
\end{aligned}
$$

We introduce the notations

$$
\begin{aligned}
I= & \int_{a}^{b} f(t) d t \\
F= & f\left(\frac{a+b}{2}\right)(b-a)+\frac{f^{\prime}(b)-f^{\prime}(a)}{24}(b-a)^{2} \\
& -\frac{1}{6} \sum_{i=2}^{m} \frac{(b-a)^{2 i+1}(2 i-2)(2 i+3)}{2^{2 i}(2 i+1) !} f^{(2 i)}\left(\frac{a+b}{2}\right) .
\end{aligned}
$$

Theorem 2.2. Let $f:[a, b] \rightarrow R$ be a function such that $f^{(n-1)}, n>3$, is absolutely continuous and there exist real numbers $\gamma_{n}, \Gamma_{n}$ such that $\gamma_{n} \leqslant f^{(n)}(t) \leqslant \Gamma_{n}, t \in[a, b]$. Then

$$
|I-F| \leqslant \frac{1}{3} \frac{\Gamma_{n}-\gamma_{n}}{(n+1) !} \frac{(n+3)(n-2)}{2^{n+2}}(b-a)^{n+1} \text { if } n \text { is odd }
$$

and

$$
|I-F| \leqslant \frac{1}{3} \frac{(n+3)(n-2)(b-a)^{n+1} n}{2^{n+1}(n+1) !}\left\|f^{(n)}\right\|_{\infty} \text { if } n \text { is even. }
$$

Proof. Let $n$ be odd. From (2) and (6) we get

$$
R(f)=(-1)^{n} \int_{a}^{b} S_{n}(t) f^{(n)}(t) d t=(-1)^{n} \int_{a}^{b} S_{n}(t)\left[f^{(n)}(t)-\frac{\gamma_{n}+\Gamma_{n}}{2}\right] d t
$$

such that we have

$$
|R(f)|=|I-F| \leqslant \max _{t \in[a, b]}\left|f^{(n)}(t)-\frac{\gamma_{n}+\Gamma_{n}}{2}\right| \int_{a}^{b}\left|S_{n}(t)\right| d t .
$$

We also have

$$
\max _{t \in[a, b]}\left|f^{(n)}(t)-\frac{\gamma_{n}+\Gamma_{n}}{2}\right| \leqslant \frac{\Gamma_{n}-\gamma_{n}}{2} .
$$

From (11), (12) and (7) we get

$$
|I-F| \leqslant \frac{1}{3} \frac{\Gamma_{n}-\gamma_{n}}{(n+1) !} \frac{n^{2}+n-6}{2^{n+2}}(b-a)^{n+1} .
$$


Let $n$ be even. Then we have

$$
|R(f)|=|I-F| \leqslant \int_{a}^{b}\left|S_{n}(t)\right| d t\left\|f^{(n)}\right\|_{\infty}=\frac{1}{3} \frac{\left(n^{2}+n-6\right)(b-a)^{n+1} n}{2^{n+1}(n+1) !}\left\|f^{(n)}\right\|_{\infty} .
$$

Theorem 2.3. Let $f:[a, b] \rightarrow R$ be a function such that $f^{(n-1)}, n>3$, is absolutely continuous and let $n$ be odd. If there exists a real number $\gamma_{n}$ such that $\gamma_{n} \leqslant f^{(n)}(t), t \in[a, b]$, then

$$
|I-F| \leqslant \frac{(n-3)(n+2)}{3}\left(T_{n}-\gamma_{n}\right) \frac{(b-a)^{n+1}}{2^{n+1}(n+1) !}
$$

where

$$
T_{n}=\frac{f^{(n-1)}(b)-f^{(n-1)}(a)}{b-a} .
$$

If there exists a real number $\Gamma_{n}$ such that $f^{(n)}(t) \leqslant \Gamma_{n}, t \in[a, b]$, then

$$
|I-F| \leqslant \frac{(n-3)(n+2)}{3}\left(\Gamma_{n}-T_{n}\right) \frac{(b-a)^{n+1}}{2^{n+1}(n+1) !}
$$

Proof. We have

$$
|R(f)|=|I-F|=\left|\int_{a}^{b}\left(f^{(n)}(t)-\gamma_{n}\right) S_{n}(t) d t\right|
$$

since (6) holds. Then we have

$$
\begin{aligned}
\left|\int_{a}^{b}\left(f^{(n)}(t)-\gamma_{n}\right) S_{n}(t) d t\right| & \leqslant \max _{t \in[a, b]}\left|S_{n}(t)\right| \int_{a}^{b}\left(f^{(n)}(t)-\gamma_{n}\right) d t \\
& =\frac{n^{2}-n-6}{3} \frac{(b-a)^{n}}{2^{n+1}(n+1) !}\left[f^{(n-1)}(b)-f^{(n-1)}(a)-\gamma_{n}(b-a)\right] \\
& =\frac{n^{2}-n-6}{3} \frac{(b-a)^{n+1}}{2^{n+1}(n+1) !}\left(T_{n}-\gamma_{n}\right) .
\end{aligned}
$$

In a similar way we can prove that (14) holds.

Remark 2.1. Note that we can apply estimations (9) and (10) only if $f^{(n)}$ is bounded. On the other hand, we can apply estimation (13) if $f^{(n)}$ is unbounded above and we can apply estimation (14) if $f^{(n)}$ is unbounded below.

\section{A numerical example}

Here we consider the integral (special function) $E i(x)=\int_{0}^{x} \frac{\exp (t)-1}{t} d t$ and apply the summation formula (1) to this integral. We get the summation formula $\operatorname{Ei}(x)=F(x)+R(x)$, 
where

$$
\begin{aligned}
F(x)= & \frac{2}{x}\left(\exp \left(\frac{x}{2}\right)-1\right)+\frac{\exp (x)(x-1)+1-\frac{x^{2}}{2}}{24} \\
& -\frac{1}{6} \sum_{i=2}^{m} \frac{(2 i-2)(2 i+3) x^{2 i+1}}{2^{2 i}(2 i+1) !} f^{(2 i)}\left(\frac{x}{2}\right)
\end{aligned}
$$

and $f(t)=\frac{\exp (t)-1}{t}, f^{\prime}(0)=\frac{1}{2}$. We calculate the derivatives $f^{(j)}(t)$ as follows. We have

$$
(g(t) h(t))^{(j)}=\sum_{k=0}^{j}\left(\begin{array}{l}
j \\
k
\end{array}\right) g^{(k)}(t) h^{(j-k)}(t) .
$$

If we choose $g(t)=\exp (t)-1$ and $h(t)=1 / t$, then we get

$$
f^{(j)}\left(\frac{x}{2}\right)=\sum_{k=0}^{j}\left(\begin{array}{c}
j \\
k
\end{array}\right)(-1)^{j-k} e_{k}\left(\frac{x}{2}\right) \frac{(j-k) ! 2^{j-k+1}}{x^{j-k+1}},
$$

where $e_{0}(t)=e^{t}-1$ and $e_{k}(t)=e^{t}, k \geqslant 1$.

We now compare the summation formula (15) with the known compound formula (for the corrected mid-point rule)

$$
\int_{0}^{x} f(t) d t=\frac{f^{\prime}(x)-f^{\prime}(0)}{24} h^{2}+h \sum_{i=0}^{n-1} f\left(\frac{x_{i}+x_{i+1}}{2}\right)+R(x),
$$

where $x_{i}=i h, h=x / n, f(t)=(\exp t-1) / t$.

Let us choose $x=1$. The "exact" value is $\operatorname{Ei}(1)=1.3179021514544$. If we use (15) with $m=5$, then we get $E i(1) \approx 1.31790215145452$. The calculations take $2.8 E-06$ seconds. If we use $(16)$ with $n=200$, then we get $E i(1) \approx 1.3179021514546$. The calculations take $4.51 E-05$ seconds. All calculations are done in double precision arithmetic. As we see, the first approximate result is obtained 16 times faster than the second one. This is a consequence of the fact that we have to calculate the function $\exp (t)$ many times when we apply the compound formula and we have only to calculate $\exp (x)$ when we apply the summation formula. (In fact, it is possible to find examples where the summation formula is a few dozen times faster than the compound formula.)

Similar summation formulas can be obtained for the integrals (special functions): $\int_{0}^{x}[(\sin t) / t] d t, \int_{0}^{x}[(\cos t-1) / t] d t, \int_{0}^{x} \exp \left(-t^{2}\right) d t$, etc.

\section{References}

[1] G. A. Anastassiou, Ostrowski type inequalities, Proc. Amer. Math. Soc., 123 (1995), no. 12, pp. 37753781.

[2] P. Cerone and S. S. Dragomir, Midpoint-type rules from an inequalities point of view, in: Handbook of Analytic-Computational Methods in Applied Mathematics (G. Anastassiou, ed.), CRC Press, New York, 2000, pp. 135-200.

[3] S. S. Dragomir, P. Cerone, and J. Roumeliotis, A new generalization of ostrowski's integral inequality for mappings whose derivatives are bounded and applications in numerical integration and for special means, Appl. Math. Lett., 13 (2000), pp. 19-25. 
[4] A. Ghizzetti and A. Ossicini, Quadrature Formulae, Birkhäuser Verlag, Basel/Stuttgart, 1970.

[5] A. R. Krommer and C. W. Ueberhuber, Computational Integration, SIAM, Philadelphia, 1998.

[6] D. S. Mitrinović, J. Pečarić, and A. M. Fink, Classical and New Inequalities in Analysis, Kluwer Acad. Publ., Dordrecht/Boston/London, 1993.

[7] C. E. M. Pearce, J. Pečarić, N. Ujević, and S. Varošanec, Generalizations of some inequalities of Ostrowski-Grüss type, Math. Inequal. Appl., 3 (2000), no. 1, pp. 25-34.

[8] N. Ujević, New bounds for the first inequality of Ostrowski-Grüss type and applications, Comput. Math. Appl., 46 (2003), pp. 421-427.

[9] N. Ujević, A generalization of Ostrowski's inequality and applications in numerical integration, Appl. Math. Lett., 17 (2004), no. 2, pp. 133-137.

[10] N. Ujević and A. J. Roberts, A corrected quadrature formula and applications, ANZIAM J., 45 (2004), no. E, pp. E41-E56. 